\title{
Medication Reconciliation Improvement Through the Use of Video
}

\author{
Maya Dewan, Blair Kraus, Daniela Davis, John McCloskey
}

The Children's Hospital of Philadelphia

\begin{abstract}
Accurate medication reconciliation at the time of hospital admission is vital to preventing adverse drug events. Compliance with medication reconciliation in our pediatric intensive care unit was low initially with overall medication reconciliation at $70 \%$. Due to the high front line provider turnover in our unit, we focused on technological reminders for completion and used unique and innovative ways to motivate our supervising staff. Our goal was to reach $>95 \%$ completion within 24 hours for medication reconciliation for all patients admitted to the pediatric intensive care unit.
\end{abstract}

Pre-pilot discussions focused on examples of errors of medication reconciliation within our own institution resulting in patient harm via traditional power point presentation. The initial pilot phase instituted a job aid on how to add the medication reconciliation completion reminder column. Email updates on completion status began one week after initiation. During the implementation and spread phase, fun interactive videos were used to acknowledge roll out to the full unit. Compliance was monitored and humorous biweekly video updates emphasizing accountability were introduced. In the sustain phase, monthly video updates served as education and a reminder for provider staff.

The use of a medication reconciliation completion column and reminder emails resulted in goal completion (>95\%) by three weeks post intervention for the pilot unit. Overall medication reconciliation completion also reached goal of $>95 \%$ completion within the entire unit after three weeks of implementation and spread. Compliance fell below goal so video updates were modified utilizing humor and emphasizing accountability. This resulted in sustained compliance now more than forty weeks post implementation.

Unit compliance currently remains $>95 \%$ completion within 24 hours now more than forty weeks post intervention. The use of the medication reconciliation reminder column resulted in improvement in compliance; however, that improvement was not sustained. The addition of humorous videos highlighting accountability allowed for sustained improvements.

\section{Problem}

Accurate medication reconciliation at the time of hospital admission is vital to preventing adverse drug events. Compliance with medication reconciliation in our pediatric intensive care unit at a large tertiary care medical center was low initially with overall hospital medication reconciliation at $70 \%$. Due to the high front line provider turn over in our unit, we focused on technological reminders for completion and used unique and innovative ways to motivate our supervising staff. Our goal was to reach $>95 \%$ completion within 24 hours for medication reconciliation by three months for all patients admitted to the pediatric intensive care unit.

\section{Background}

Accurate medication reconciliation at the time of hospital admission is vital to preventing adverse drug events. At the time of admission, one quarter of all patients in one study had at least one medication error when reviewed by a multi-disciplinary team.[1] This risk of medication error has been found to be even higher for patients in the intensive care unit [2] and pediatric patients are three times more likely to experience medication errors with the potential to cause harm [3]. These adverse drug events carry high cost [4] and result in increased patient morbidity and mortality. However, despite these known facts, medication reconciliation remains difficult and compliance levels low.

\section{Baseline measurement}

A retrospective audit of compliance was obtained through the electronic medical record system for all patients admitted to the pediatric intensive care unit at The Children's Hospital of Philadelphia. Compliance with medication reconciliation in our pediatric intensive care unit was low initially with overall medication reconciliation at $70 \%$.

See supplementary file: ds4592.png - "Figure 1: Pilot data and baseline measurement"

\section{Design}

Specific workflow through the electronic medical record already existed for medication reconciliation at our institution. Pre-pilot discussions focused on examples of errors of medication reconciliation within our own institution resulting in patient harm. This was done via a traditional PowerPoint presentation that was shown to front line care providers and supervisory physicians in our unit.

Due to the high front line provider turnover in our unit, we focused 
on technological reminders including a job aid and addition of a medication reconciliation reminder column. The job aid focused on how to add the medication reconciliation completion reminder column and was posted at front line clinician work desks. Email updates on completion status began one week after initiation of the column and percentage of medication reconciliation competition within the pilot unit was compared to the control units.

\section{Strategy}

Fun interactive videos were used to acknowledge roll out to full unit during the implementation and spread phase. Job aids were posted throughout the remainder of the unit and email updates were augmented with video clips. Compliance was monitored and humorous biweekly video updates emphasizing accountability were introduced to help sustain improvement (go to

http://youtu.be/NaTO1DG1oql for one example). During the sustain phase, monthly video updates served as education and a reminder for provider staff (go to http://youtu.be/zVZmERIxT3k for an example of a sustain phase video).

\section{Results}

The use of medication reconciliation completion column and reminder emails resulted in goal completion $(>95 \%)$ by three weeks post intervention for the pilot unit. Overall medication reconciliation completion also reached the goal of $>95 \%$ completion within the entire unit after three weeks of implementation and spread.

Compliance fell below goal so video updates were modified utilizing humor and emphasizing accountability. This resulted in sustained compliance now more than forty weeks post intervention.

See supplementary file: ds4566.docx - "Figure 2: Implementation and Spread Phase throughout the PICU"

\section{Lessons and limitations}

During implementation of this project, it became immediately clear that despite the known safety benefits to proper medication reconciliation, these benefits were under appreciated by our front line providers. Initial education focused on errors within our own institution due to improper medication reconciliation. While small improvements were noted after this education, sustained improvement required the key supervisory stakeholders to become part of the process. With the additional of attending and fellow involvement through the use of the medication reconciliation reminder column, compliance improved. However, it took the unique and innovative approach of video updates to change our culture and make medication reconciliation a recognized approach to improving patient safety.

This study focused only on completion of medication reconciliation as the end point. Further work must be completed focusing on medication reconciliation accuracy improvements.
Unit compliance currently remains $>95 \%$ completion within 24 hours now more than forty weeks post intervention. The use of the medication reconciliation reminder column resulted in improvement in compliance; however, that improvement was not sustained. Addition of humorous videos highlighting accountability allowed for sustained improvements. Future work on medication reconciliation accuracy are in development at our institution and will serve to further improve safety of our patients and decrease medication administration errors.

\section{References}

1. Gleason KM, Groszek JM, Sullivan C, Rooney D, Barnard C, Noskin GA. Reconciliation of discrepancies in medication histories and admission orders of newly hospitalized patients. Am J Health Syst Pharm 2004;61(16):1689-94.

2. Bell CM, Brener SS, Gunraj N, et al. Association of ICU or hospital admission with unintentional discontinuation of medications for chronic diseases. JAMA 2011;306(8):840-7.

3. Fortescue EB, Kaushal R, Landrigan CP, et al. Prioritizing strategies for preventing medication errors and adverse drug events in pediatric inpatients. Pediatrics 2003;111(3):722-9.

4. Bates DW, Spell N, Cullen DJ, et al. The costs of adverse drug events in hospitalized patients. Adverse Drug Events Prevention Study Group. JAMA 1997;277(4):307-11.

\section{Declaration of interests}

Nothing to declare.

\section{Acknowledgements}

Amina Khan, Heather Wolfe 